\title{
Cavity-Enhanced Real-Time Monitoring of Single-Charge Jumps at the Microsecond Time Scale
}

\author{
C. Arnold, ${ }^{1}$ V. Loo, ${ }^{1,2}$ A. Lemaître, ${ }^{1}$ I. Sagnes, ${ }^{1}$ O. Krebs, ${ }^{1}$ P. Voisin,,${ }^{1}$ P. Senellart, ${ }^{1}$ and L. Lanco ${ }^{1,2, *}$ \\ ${ }^{1}$ Laboratoire de Photonique et Nanostructures, LPN/CNRS, Route de Nozay, 91460 Marcoussis, France \\ ${ }^{2}$ Département de Physique, Université Paris Diderot, 4 rue Elsa Morante, 75013 Paris, France \\ (Received 22 April 2013; revised manuscript received 22 February 2014; published 4 April 2014)
}

\begin{abstract}
We use fast coherent reflectivity measurements, in a strongly coupled quantum dot micropillar device, to monitor in real time single-charge jumps at the microsecond time scale. Thanks to the strong enhancement of light-matter interaction inside the cavity, and to a close to shot-noise-limited detection setup, the measurement rate is 5 orders of magnitude faster than with previous optical experiments of direct single-charge sensing with quantum dots. The monitored transitions, identified at any given time with a less than $0.2 \%$ error probability, correspond to a carrier being captured and then released by a single material defect. This high-speed technique opens the way for the real-time monitoring of other rapid single quantum events, such as the quantum jumps of a single spin.
\end{abstract}

DOI: 10.1103/PhysRevX.4.021004

Semiconductor quantum dots (QDs) have attracted considerable interest as important building blocks for quantum information experiments based on photon qubits [1], exciton qubits [2], or spin qubits [3]. The scalability of this solid-state implementation of a quantum network relies on the possibility of building a highly efficient single QDsingle photon interface [4]. For the photon qubits, the challenge consists in collecting all single photons emitted by the QDs. For the exciton or spin qubits, the challenge is to strongly increase the light-matter interaction, so that a single photon sent on the device will significantly modify the system state and its optical response [5]. In the past decade, cavity quantum electrodynamics has been shown to constitute a powerful tool to build such a QD-photon interface. Inserting a QD in a pillar cavity has allowed the collection of around $80 \%$ of the single photons emitted by a QD [6], as well as the demonstration of optical nonlinearities for a coherent beam containing as few as 8 photons per pulse [7]. The exciton qubit in a photonic crystal cavity has also been used to implement ultrafast optical switches [8-10] and logic gates at the few photon scale [11]. Last but not least, coupling a charged quantum dot to a cavity has been proposed for single spin nondestructive measurement, remote spin entanglement, and entanglement between delayed photons [5,12].

In this paper, we show that a highly efficient QD-cavity interface makes it possible to monitor in real time single quantum events at the microsecond time scale. This is

\footnotetext{
*loic.lanco@lpn.cnrs.fr
}

Published by the American Physical Society under the terms of the Creative Commons Attribution 3.0 License. Further distribution of this work must maintain attribution to the author(s) and the published article's title, journal citation, and DOI.
Subject Areas: Semiconductor Physics

illustrated here by monitoring in real time single-charge jumps, evidencing a measurement rate 5 orders of magnitude faster than for previous optics experiments of direct single-charge sensing [13-18]. Our technique relies on coherent reflection spectroscopy, performed with a detection setup approaching the shot-noise limit, on a deterministically coupled QD-pillar cavity device, into which the incident photons are injected with a high input-coupling efficiency [7]. This ensures that almost every incident photon interacts with the QD and provides an optical response highly sensitive to the QD transition energy. Single events, corresponding to the capture and release of a single charge by a material defect, are distinctly identified with a few microseconds time resolution and with a less than $0.2 \%$ error probability. Our measurements also reveal a photoinduced acceleration of the charge dynamics. This technique could be used to monitor other rapid single events such as spin flips of a single electron or hole, typically occurring between the microsecond and millisecond time scales [19].

The energy of a QD transition is highly sensitive to fluctuations of the local electric field induced by the random motion of a few charges around the QD [20-22], leading to pure dephasing or inhomogeneous broadening of the transition linewidth [23]. Discrete spectral QD fluctuations have been reported at the few seconds or minutes time scale, monitoring the escape or capture of charges from material defects around a QD [13-18]. Here, we investigate a simple case where the fluctuation of a single charge around a QD makes the QD exciton energy $\omega_{d}$ switch between two discrete different values: $\omega_{d}^{(L)}$ if a nearby material defect is loaded by a trapped carrier [Fig. 1(a)] and $\omega_{d}^{(E)}$ if this defect is empty [Fig. 1(b)]. Capture and release of a carrier are, therefore, detected through the modification of $\omega_{d}$. 
(a)

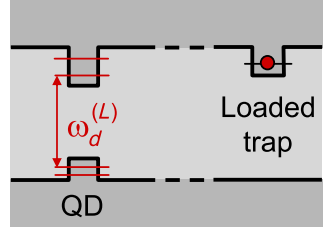

(b)

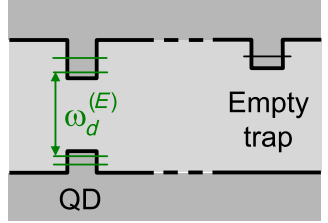

(d)
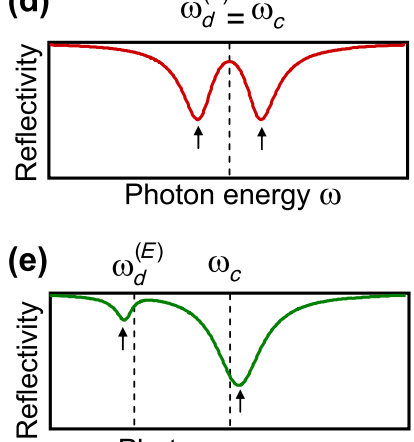

Photon energy $\omega$

(c) $2 \mathrm{MHz}$

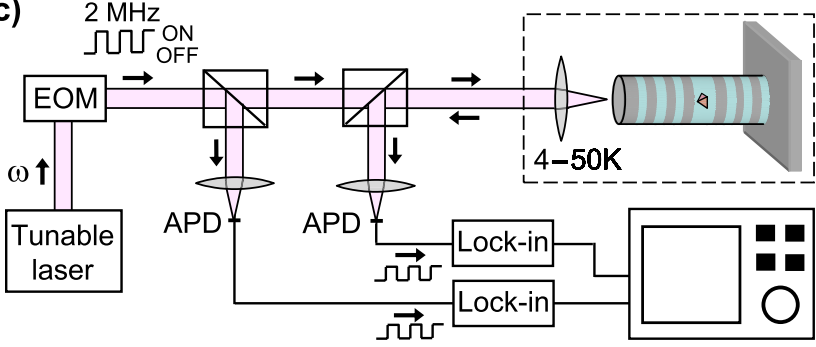

FIG. 1. (a),(b) Band structures of an InGaAs QD with a nearby loaded or empty trap, corresponding to QD transition energies $\omega_{d}^{(L)}$ and $\omega_{d}^{(E)}$. (c) Experimental setup (EOM, electro-optic modulation system; APD, avalanche photodiodes). (d),(e) Typical reflectivity spectra for a loaded and for an empty trap, in the special case where $\omega_{d}^{(L)}=\omega_{c}$ but $\omega_{d}^{(E)}<\omega_{c}$. The vertical arrows underline the energies of the mixed exciton-photon eigenstates.

To greatly enhance the sensitivity to these fluctuations, the QD line is coupled to a pillar cavity in the strong coupling regime [24]. The sample consists in a single InGaAs QD deterministically coupled to a pillar microcavity using the in situ lithography technique $[25,26]$.

The experimental setup is described in Fig. 1(c). The sample is placed inside a helium vapor cryostat, together with a focusing lens and three nanopositioners for the optical alignment. A continuous-wave monomode laser, with a finely tunable photon energy $\omega$, is focused on and reflected from the micropillar. The incident and reflected powers are measured with fast silicon avalanche photodiodes. Each photodiode is connected to a lock-in amplifier, while the intensity of the incident laser is chopped at $2 \mathrm{MHz}$ frequency with an electro-optic modulation system. This allows the lock-in measurement of both signals with an integration time down to $2 \mu \mathrm{s}$. The experimental reflectivity is computed as the ratio between reflected power and incident power, and then normalized: it tends towards unity far from the cavity resonance [7]. The setup ensures a near-unity input-coupling efficiency of the incident photons into the micropillar fondamental mode $[7,27]$ and a very high mechanical stability during tens of hours. In typical experimental conditions, the measured detection noise is around twice the ideal shot-noise limit [24].
In the absence of light-matter coupling, a cavity reflectivity spectrum presents only one Lorentzian dip, at the bare cavity mode resonance energy $\omega_{c}$. In the strong coupling regime, the reflectivity spectrum of a QD-cavity device presents two dips at the eigenenergies of the coupled system [28]. Figure 1(d) displays a sketch of a typical reflectivity spectrum, as a function of the laser photon energy $\omega$, in a configuration where the trap is loaded and where $\omega_{d}^{(L)}=\omega_{c}$. In such a case, both eigenstates have equal photonic and excitonic parts and are symmetrically detuned from $\omega_{d}^{(L)}=\omega_{c}$. When the trap is empty, the bare QD transition energy becomes $\omega_{d}^{(E)}$, whereas the bare cavity mode energy $\omega_{c}$ remains unchanged. As illustrated in Fig. 1(e), if $\omega_{d}^{(E)}$ is lower than $\omega_{c}$, an assymetrical spectrum is obtained where one eigenstate is mainly excitonlike (energy close to $\omega_{d}^{(E)}$ ) and the other one is mainly photoniclike (energy close to $\omega_{c}$ ).

Figure 2(a) presents a scatter plot of several reflectivity values measured as a function of the photon energy $\omega$ for an incident power $P_{0}=1.7 \mathrm{nW}$ and a temperature $T=34.1 \mathrm{~K}$. It consists of $10^{4}$ reflectivity measurements, each with a $50 \mu$ s integration time; a $1 \mathrm{~ms}$ delay between measurements has been introduced to ensure that two consecutive measurements are uncorrelated. This plot highlights the presence of strong reflectivity fluctuations that cannot be accounted for by the experimental noise. The solid curves in this figure are numerical fits obtained with a single set of device parameters [24], only differing by the values of $\omega_{d}^{(E)}$ and $\omega_{d}^{(L)}$ as in Figs. 1(d) and 1(e). The fitted
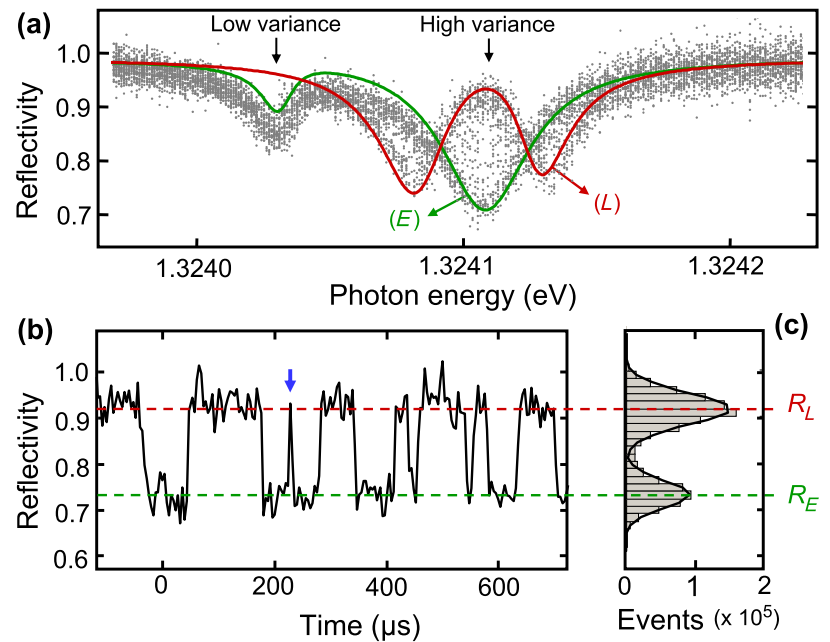

FIG. 2. (a) Scatter plot of measured reflectivity values versus photon energy at $T=34.1 \mathrm{~K}$ and $P_{0}=1.7 \mathrm{nW}$. Solid lines: theoretical fit. (b) Real-time reflectivity signal at $T=34.1 \mathrm{~K}$, $P_{0}=5.5 \mathrm{nW}$, and $\omega=1.32411 \mathrm{eV}$. Dashed horizontal lines are guides to the eye indicating the two states with reflectivities $R_{L}$ and $R_{E}$. (c) Histogram of the reflectivity values measured in the experimental conditions of Fig. 2(b). Solid line: numerical fit with a sum of two Gaussian distribution functions centered around $R_{L}$ and $R_{E}$. 
energy difference, $\omega_{d}^{(E)}-\omega_{d}^{(L)}=75 \pm 5 \mu \mathrm{eV}$, is too low to be compatible with a charge fluctuation in the quantum dot itself, but is compatible with a nearby material defect, at a distance of several tens of nanometers from the quantum dot, randomly capturing and releasing single charges $[18,21]$. The fact that different reflectivity variances are observed in different regions of the spectrum is discussed below.

To perform the real-time monitoring of capture and release events, we tune the laser to $\omega=1.32411 \mathrm{eV}$, corresponding to the region of very high variance highlighted in Fig. 2(a). Figure 2(b) displays a typical real-time reflectivity measurement in this configuration, obtained with a $2 \mu$ s integration time: the signal-to-noise ratio is high enough to allow the direct observation of random jumps between two reflectivity values, $R_{E}$ and $R_{L}$. These jumps are observed each time the system experiences an $E \rightarrow L$ transition (capture) or an $L \rightarrow E$ transition (release). As an example, the narrow reflectivity peak emphasized in Fig. 2(b) corresponds to the capture and, approximately $6 \mu$ s later, to the consecutive release of a single charge.

The clear distinction between the loaded and empty states is also illustrated in the reflectivity histogram of Fig. 2(c), obtained from a set of $10^{6}$ real-time measurements with a $2 \mu$ s integration time. This histogram is well reproduced numerically with a sum of two Gaussian distribution functions centered at $R_{E}=0.72$ and $R_{L}=0.93$. The overlap between the two distributions is small enough to allow us, at any time and with a less than $0.2 \%$ error probability, to identify whether the system is in the state $E$ or $L$ [24]. Furthermore, we can measure with a few microseconds precision the time at which the system undergoes an $L \rightarrow E$ or an $E \rightarrow L$ transition, and, thus, the time spent in the state $E$, or $L$, between two transitions.

Figure 3(a) displays, for different excitation powers, experimental histograms of the time spent by the system in state $E$ before experiencing an $E \rightarrow L$ transition; as in Fig. 2(c), these histograms are extracted with sets of $10^{6}$ real-time measurements. In each case, the probability that the system remains in state $E$ exponentially decreases with the elapsed time; the corresponding monoexponential law is characterized by a negative slope, whose absolute value gives the transition rate from the empty state to the loaded one, denoted $\Gamma_{E \rightarrow L}$. The four histograms in Fig. 3(a) have been acquired for various values of the excitation power $P_{0}$, showing that $\Gamma_{E \rightarrow L}$ increases with the incident power. The same set of real-time reflectivity measurements has been used to extract similar histograms [24] of the time spent in the loaded state $L$, before experiencing a $L \rightarrow E$ transition: monoexponential decreases are also observed, with a transition rate denoted $\Gamma_{L \rightarrow E}$ that also increases with the incident power. Even if it is unexpected under resonant excitation, the only explanation of this acceleration with the incident power is that a photoinduced carrier generation occurs in the InAs wetting layer or in the GaAs barriers.
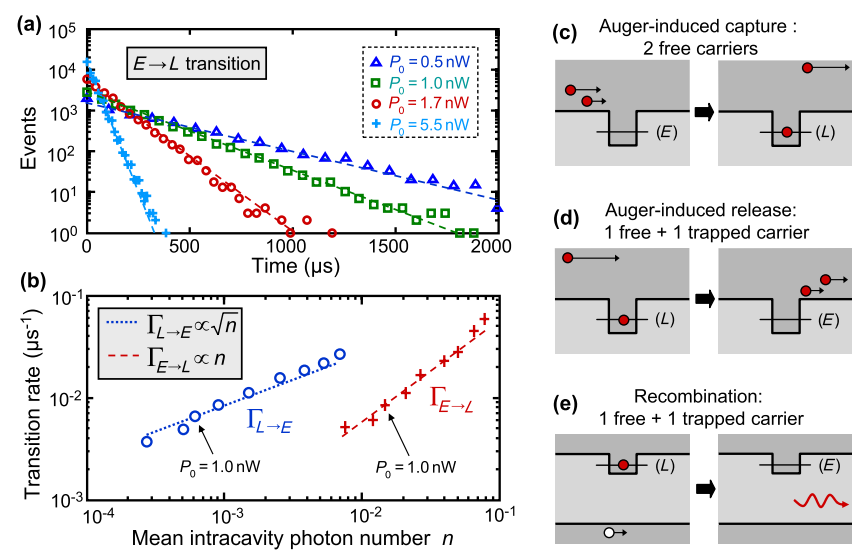

(d) Auger-induced release:
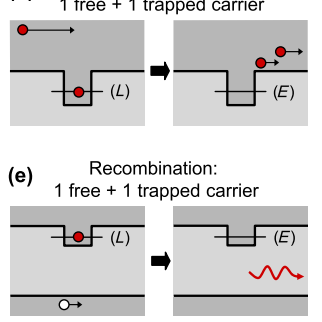

FIG. 3. (a) Histogram of the time spent in state $E$ before returning to state $L$, at $T=34.1 \mathrm{~K}$ and $\omega=1.32411 \mathrm{eV}$, for various excitation powers. Dashed lines: numerical fits for monoexponential decays, from which the transition rates $\Gamma_{E \rightarrow L}$ are extracted. (b) Measured transition rates $\Gamma_{E \rightarrow L}$ and $\Gamma_{L \rightarrow E}$, extracted from the real-time measurements, as a function of the intracavity photon number $n$. Two different photon numbers are considered for each value of the incident power $P_{0}$ : the photon number in the $E$ state governs the $\Gamma_{E \rightarrow L}$ transition rate, while the photon number in the $L$ state governs the $\Gamma_{L \rightarrow E}$ transition rate. Dashed and dotted lines: numerical fits (see legend). (c)-(e) Schematic view of some microscopic processes allowing for capture and release events.

Because the resonant photon energy is lower than the energy required for direct interband transition, this generation is probably mediated by the interaction with phonons or with carriers already present in the material. It could also be assisted by the presence of discrete states in neighboring material defects or quantum dots. We point out that sub-band-gap absorption, leading to charge fluctuations stemming from photoexcited free carriers, has already been reported in a cavity-QED device [29].

The relevant quantity, for the analysis of this photoinduced acceleration of the fluctuations, is not the incident power but the number $n$ of intracavity photons, which have a nonzero probability of exciting an electron-hole pair. The generated carriers can then participate in the $E \rightarrow L$ transition, if the system is initially in state $E$, or in the $L \rightarrow E$ transition, if the system is initially in state $L$. The intracavity photon number $n$ takes the form $n \propto P_{0}\left|1-r_{m}\right|^{2}$, where $r_{m}$ is the mode reflection coefficient [24]; $r_{m}$ and, thus, $n$, take different values if the device is in state $E$ or $L$. Figure 3(b) shows the transition rates $\Gamma_{E \rightarrow L}$ and $\Gamma_{L \rightarrow E}$, as a function of $n$. For a real-time measurement at a given incident power $P_{0}$, the value of $n$ considered is the one in state $E$ for the $E \rightarrow L$ transition, and the one in state $L$ for the $L \rightarrow E$ transition.

The data in Fig. 3(b) allow one to deduce the empiric laws governing the increase of the transition rates with $n$. A satisfying fit of the experimental data is obtained with two different laws, namely, 


$$
\Gamma_{E \rightarrow L}=\alpha n \quad \text { and } \quad \Gamma_{L \rightarrow E}=\beta \sqrt{n},
$$

where $\alpha=0.6 \mu \mathrm{s}^{-1}$ and $\beta=0.27 \mu \mathrm{s}^{-1}$ are empirical proportionality coefficients. Even if it is beyond the scope of this paper to identify which physical process is responsible for the carrier generation, we show below that a simple model allows us to understand why, if a carrier generation does occur, it can be expected to lead to such linear and sublinear behaviors with the number of internal photons. Following previous works [23,30-33], we consider that the carrier generation rate is proportional to $n$, while the carrier recombination rate is proportional to $N_{e} N_{h}=N_{c}^{2}$, where $N_{e}=N_{h}$ are the electron and hole densities, considered both equal to the carrier density denoted $N_{c}$. In this model, where $d N_{c} / d t=a n-b N_{c}^{2}, a$ and $b$ being constant proportionality coefficients, the stationary regime $d N_{c} / d t=0$ corresponds to a carrier density $N_{c}$ proportional to $\sqrt{n}$. The empiric power laws deduced from our real-time measurements can thus be rewritten in the simple forms $\Gamma_{E \rightarrow L} \propto N_{c}^{2}$ and $\Gamma_{L \rightarrow E} \propto N_{c}$.

As we now describe, such power laws find a direct interpretation within a simple model where capture and release events require the interaction between two carriers in the vicinity of the defect. For example, as illustrated in Fig. 3(c), an Auger capture process involves an inelastic collision between two free carriers near the material defect, one of which ends up captured in the trap: the expected interaction rate is then proportional to the square of the carrier density, in agreement with the power law $\Gamma_{E \rightarrow L} \propto N_{c}^{2}$. Conversely, as illustrated in Fig. 3(d), the release of the trapped carrier can also result from an Auger inelastic collision between the trapped carrier and an incoming free carrier; the expected interaction rate is proportional to the carrier density, in agreement with the linear law $\Gamma_{L \rightarrow E} \propto N_{c}$. Another physical process compatible with this law is when the interaction between the trapped carrier and an incoming free carrier leads to the recombination of both, as illustrated in Fig. 3(e); this again gives a transition rate $\Gamma_{L \rightarrow E} \propto N_{c}$. We point out that Nguyen et al., to numerically reproduce their experimental data, have recently used a model with power laws similar to those of Eq. (1) [33]. Because of the aboveband-gap excitation scheme in their experiment, short transition times were observed, down to the nanosecond time scale. The typical transition times in our experiment are several orders of magnitude higher, approaching the millisecond time scale at the lowest incident powers. This is fully consistent with the fact that, for a given incident power, above-band-gap excitation will induce a much higher number of photocreated carriers, compared to our resonant excitation scheme.

We now show that the measured empiric laws of Eq. (1) are valid for a large range of experimental parameters, by performing measurements with various device temperatures and laser wavelengths. Indeed, as the temperature interval for which the fluctuations are clearly observed is a
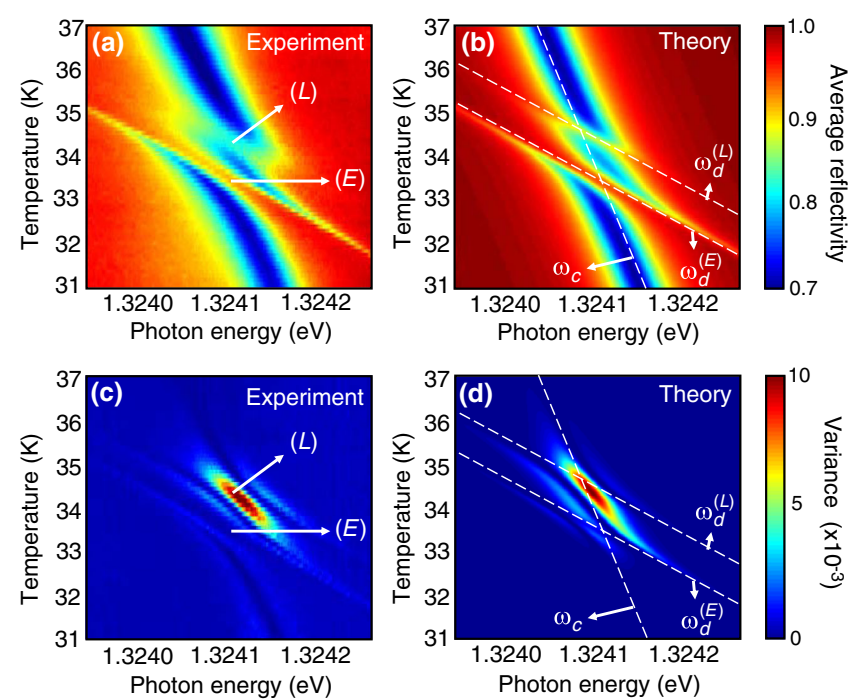

FIG. 4. (a) Experimental average reflectivity versus temperature and photon energy. (b) Simulated average reflectivity. (c) Experimental reflectivity variance versus temperature and photon energy. (d) Simulated reflectivity variance. The dashed lines in the simulated maps indicate the bare cavity mode and QD transition energies. The arrows in the experimental maps indicate where the anticrossings occur, which is when $\omega=\omega_{d}^{(L)}=\omega_{c}$ (for state $L$ ) or when $\omega=\omega_{d}^{(E)}=\omega_{c}$ (for state $E$ ).

few kelvins wide, no significant deviation from the laws of Eq. (1) is expected, neither in the form of the equation nor in the value of the $\alpha$ and $\beta$ coefficients. When tuning the device temperature, we simply introduce a continuous change in the bare cavity mode energy $\omega_{c}$ at a rate of $17 \mu \mathrm{eV} / \mathrm{K}$, and in the bare QD transition energies $\omega_{d}^{(L)}$ and $\omega_{d}^{(E)}$ at a rate of $81 \mu \mathrm{eV} / \mathrm{K}$ [28], with a constant energy shift $\omega_{d}^{(E)}-\omega_{d}^{(L)}=75 \mu \mathrm{eV}$. For each set of experimental conditions, we record 10000 successive measurements with a $2 \mu$ s integration time; the experimental reflectivity average $R_{\mathrm{av}}$ and variance $\sigma_{R}^{2}$ are then extracted from these data. Figure 4(a) first displays in color scale the experimental average reflectivity, as a function of temperature and photon energy $\omega$, for an incident power $P_{0}=1.7 \mathrm{nW}$. Two anticrossings are observed with the cavity resonance, associated with the $E$ and $L$ states, instead of only one anticrossing for a nonfluctuating device [7].

To predict the theoretical average and variance of the reflectivity signal, the parameters already used in the fits of Fig. 2(a) are applied, without further adjustment: this allows us to deduce the reflectivities for each state, $R_{E}$ and $R_{L}$, and the corresponding photon numbers in the two states $E$ and $L$. The theoretical transition rates $\Gamma_{E \rightarrow L}$ and $\Gamma_{L \rightarrow E}$ are then computed using the power laws in Eq. (1), and the overall probabilities of the system being in the empty or loaded state, $P_{E}$ and $P_{L}$, are calculated using $P_{E}=\Gamma_{L \rightarrow E} /\left(\Gamma_{L \rightarrow E}+\Gamma_{E \rightarrow L}\right)$ and $P_{L}=1-P_{E}$ [24]. The theoretical average reflectivity $R_{\mathrm{av}}=P_{E} R_{E}+P_{L} R_{L}$ is plotted in Fig. 4(b), showing good agreement with the 
experimental data. As a guide to the eyes, the values of the bare cavity mode frequency $\omega_{c}$ and the bare QD transition frequencies $\omega_{d}^{(E)}$ and $\omega_{d}^{(L)}$ are indicated: the anticrossings occur for $\omega_{c} \approx \omega_{d}^{(E)}$ and $\omega_{c} \approx \omega_{d}^{(L)}$.

A fair agreement is also obtained between the experimental and calculated variance color maps, displayed, respectively, in Figs. 4(c) and 4(d). In the latter case, the theoretical variance is given by $\sigma_{R}^{2}=P_{E} P_{L}\left(R_{E}-R_{L}\right)^{2}$ : a high variance $\sigma_{R}^{2}$ thus requires different reflectivities $R_{E}$ and $R_{L}$, but also comparable probabilities $P_{E}$ and $P_{L}$. Only one region of high variance is obtained, around the loadedstate anticrossing, where there is a maximal reflectivity difference $R_{L}-R_{E}$ and where the power laws of Eq. (1) lead to comparable probabilities $P_{L} \approx P_{E}$. Almost no variance is observed, on the contrary, near the empty-state anticrossing where Eq. (1) leads to $P_{L} \ll P_{E}$ [24]. The high-variance and low-variance regions previously highlighted in Fig. 2(a) correspond to similar situations where $P_{L} \approx P_{E}$ and $P_{L} \ll P_{E}$, respectively. Note that, with a photoluminescence experiment using above-band excitation, only one of the two states is observed; this further illustrates that the relative occupancy of these states strongly depends on the excitation conditions.

In conclusion, we show that cavity-enhanced coherent reflectivity measurements allow the real-time monitoring of single-charge jumps at the microsecond time scale. The capture and release of a charge by a material defect has been directly observed in a strongly coupled QD-cavity system, highly sensitive to single-carrier fluctuations in the QD environment. All the experimental observations allow us to propose an empiric model, where the fluctuation processes are governed by the interaction between two carriers in the vicinity of the defect. Our results also evidence the backaction of the measurement on the system dynamics, as free carriers are generated in the wetting layer or in the GaAs barriers in spite of the purely resonant excitation scheme. Fast coherent measurements in optical microcavities can also be extended to the real-time monitoring of a single electron or hole spin in a charged quantum dot. This would constitute a quantum nondemolition experiment, one of the fundamental building blocks of a spin-photon interface, where the spin state is projected by the measurement onto one of its two possible eigenstates.

We thank H. S. Nguyen and I. Favero for fruitful discussions. This work was partially supported by the French ANR MIND, ANR CAFE, ANR QDOM, the ERC starting Grant No. 277885 QD-CQED, the CHISTERA project SSQN, and the French RENATECH network.

[1] A. J. Shields, Semiconductor Quantum Light Sources, Nat. Photonics 1, 215 (2007).

[2] E. Poem, Y. Kodriano, C. Tradonsky, N. H. Lindner, B. D. Gerardot, P. M. Petroff, and D. Gershoni, Accessing the Dark Exciton with Light, Nat. Phys. 6, 993 (2010).
[3] R. Hanson, V. V. Dobrovitski, A. E. Feiguin, O. Gywat, and D. D. Awschalom, Coherent Dynamics of a Single Spin Interacting with an Adjustable Spin Bath, Science 320, 352 (2008).

[4] H. J. Kimble, The Quantum Internet, Nature (London) 453, 1023 (2008).

[5] C. Y. Hu, A. Young, J. L. O'Brien, W. J. Munro, and J. G. Rarity, Giant Optical Faraday Rotation Induced by a Single-Electron Spin in a Quantum Dot: Applications to Entangling Remote Spins via a Single Photon, Phys. Rev. B 78, 085307 (2008).

[6] O. Gazzano, S. M. de Vasconcellos, C. Arnold, A. Nowak, E. Galopin, I. Sagnes, L. Lanco, A. Lemaître, and P. Senellart, Bright Solid-State Sources of Indistinguishable Single Photons, Nat. Commun. 4, 1425 (2013).

[7] V. Loo, C. Arnold, O. Gazzano, A. Lemaître, I. Sagnes, O. Krebs, P. Voisin, P. Senellart, and L. Lanco, Optical Nonlinearity for Few-Photon Pulses on a Quantum DotPillar Cavity Device, Phys. Rev. Lett. 109, 166806 (2012).

[8] T. Volz, A. Reinhard, M. Winger, A. Badolato, K. J. Hennessy, E. L. Hu, and A. Imamoglu, Ultrafast All-Optical Switching by Single Photons, Nat. Photonics 6, 607 (2012).

[9] R. Bose, D. Sridharan, H. Kim, G. S. Solomon, and E. Waks, Low-Photon-Number Optical Switching with a Single Quantum Dot Coupled to a Photonic Crystal Cavity, Phys. Rev. Lett. 108, 227402 (2012).

[10] D. Englund, A. Majumdar, M. Bajcsy, A. Faraon, P. Petroff, and J. Vuckovic, Ultrafast Photon-Photon Interaction in a Strongly Coupled Quantum Dot-Cavity System, Phys. Rev. Lett. 108, 093604 (2012).

[11] H. Kim, R. Bose, T. C. Shen, G. S. Solomon, and E. Waks, A Quantum Logic Gate between a Solid-State Quantum Bit and a Photon, Nat. Photonics 7, 373 (2013).

[12] C. Bonato, F. Haupt, S. S. R. Oemrawsingh, J. Gudat, D. Ding, M. P. van Exter, and D. Bouwmeester, CNOT and Bell-State Analysis in the Weak-Coupling Cavity QED Regime, Phys. Rev. Lett. 104, 160503 (2010).

[13] S. A. Empedocles, D. J. Norris, and M. G. Bawendi, Photoluminescence Spectroscopy of Single CdSe Nanocrystallite Quantum Dots, Phys. Rev. Lett. 77, 3873 (1996).

[14] H. D. Robinson and B. B. Goldberg, Light-Induced Spectral Diffusion in Single Self-Assembled Quantum Dots, Phys. Rev. B 61, R5086 (2000).

[15] M. Sugisaki, H.-W. Ren, K. Nishi, and Y. Masumoto, Fluorescence Intermittency in Self-assembled InP Quantum Dots, Phys. Rev. Lett. 86, 4883 (2001).

[16] L. Besombes, K. Kheng, L. Marsal, and H. Mariette, Few-Particle Effects in Single CdTe Quantum Dots, Phys. Rev. B 65, 121314 (2002).

[17] A. Majumdar, E. D. Kim, and J. Vuckovic, Effect of Photogenerated Carriers on the Spectral Diffusion of a Quantum Dot Coupled to a Photonic Crystal Cavity, Phys. Rev. B 84, 195304 (2011).

[18] J. Houel, A. V. Kuhlmann, L. Greuter, F. Xue, M. Poggio, B. D. Gerardot, P. A. Dalgarno, A. Badolato, P. M. Petroff, A. Ludwig, D. Reuter, A. D. Wieck, and R. J. Warburton, Probing Single-Charge Fluctuations at a GaAs/AlAs Interface Using Laser Spectroscopy on a Nearby InGaAs Quantum Dot, Phys. Rev. Lett. 108, 107401 (2012). 
[19] M. Kroutvar, Y. Ducommun, D. Heiss, M. Bichler, D. Schuh, G. Abstreiter, and J. J. Finley, Optically Programmable Electron Spin Memory Using Semiconductor Quantum Dots, Nature (London) 432, 81 (2004).

[20] B. Alen, F. Bickel, K. Karrai, R. J. Warburton, and P. M. Petroff, Stark-Shift Modulation Absorption Spectroscopy of Single Quantum Dots, Appl. Phys. Lett. 83, 2235 (2003).

[21] A. N. Vamivakas, Y. Zhao, S. Fält, A. Badolato, J. M. Taylor, and M. Atatüre, Nanoscale Optical Electrometer, Phys. Rev. Lett. 107, 166802 (2011).

[22] A. V. Kuhlmann, J. Houel, A. Ludwig, L. Greuter, D. Reuter, A. D. Wieck, M. Poggio, and R. J. Warburton, Charge Noise and Spin Noise in a Semiconductor Quantum Device, Nat. Phys. 9, 570 (2013).

[23] A. Berthelot, I. Favero, G. Cassabois, C. Voisin, C. Delalande, P. Roussignol, R. Ferreira, and J. M. Gérard, Unconventional Motional Narrowing in the Optical Spectrum of a Semiconductor Quantum Dot, Nat. Phys. 2, 759 (2006).

[24] See Supplemental Material at http://link.aps.org/ supplemental/10.1103/PhysRevX.4.021004 for details on the sample structure and experimental analyses.

[25] A. Dousse, L. Lanco, J. J. Suffczynski, E. Semenova, A. Miard, A. Lemaitre, I. Sagnes, C. Roblin, J. Bloch, and P. Senellart, Controlled Light-Matter Coupling for a Single Quantum Dot Embedded in a Pillar Microcavity Using Far-Field Optical Lithography, Phys. Rev. Lett. 101, 267404 (2008).

[26] A. Dousse, J. Suffczynski, R. Braive, A. Miard, A. Lemaître, I. Sagnes, L. Lanco, J. Bloch, P. Voisin, and P. Senellart, Scalable Implementation of Strongly Coupled
Cavity-Quantum Dot Devices, Appl. Phys. Lett. 94, 121102 (2009).

[27] C. Arnold, V. Loo, A. Lemaître, I. Sagnes, O. Krebs, P. Voisin, P. Senellart, and L. Lanco, Optical Bistability in a Quantum Dots/Micropillar Device with a Quality Factor Exceeding 200 000, Appl. Phys. Lett. 100, 111111 (2012).

[28] V. Loo, L. Lanco, A. Lemaître, I. Sagnes, O. Krebs, P. Voisin, and P. Senellart, Quantum Dot-Cavity StrongCoupling Regime Measured through Coherent Reflection Spectroscopy in a Very High-Q Micropillar, Appl. Phys. Lett. 97, 241110 (2010).

[29] K. Srinivasan, C. P. Michael, R. Perahia, and O. Painter, Investigations of a Coherently Driven Semiconductor Optical Cavity QED System, Phys. Rev. A 78, 033839 (2008).

[30] J. E. Caroll, Rate Equation in Semiconductor Electronics (Cambridge University Press, Cambridge, England, 1985).

[31] K. E. O'Hara, J. R. Gullingsrud, and J. P. Wolfe, Auger Decay of Excitons in $\mathrm{Cu}_{2} \mathrm{O}$, Phys. Rev. B 60, 10872 (1999).

[32] G. Sallen, A. Tribu, T. Aichele, R. André, L. Besombes, C. Bougerol, M. Richard, S. Tatarenko, K. Kheng, and J.-P. Poizat, Subnanosecond Spectral Diffusion of a Single Quantum Dot in a Nanowire, Phys. Rev. B 84, 041405 (2011).

[33] H. S. Nguyen, G. Sallen, M. Abbarchi, R. Ferreira, C. Voisin, P. Roussignol, G. Cassabois, and C. Diederichs, Photoneutralization and Slow Capture of Carriers in Quantum Dots Probed by Resonant Excitation Spectroscopy, Phys. Rev. B 87, 115305 (2013). 